\title{
Análise da prescrição de radiografias por acadêmicos de Odontologia de uma universidade pública brasileira e desenvolvimento de um modelo didático
}

\author{
Rafael Mendes Del Queiroz*; Wellen Góbi Botacin*; Adriana Lira Ortega**; Mariana Ferreira \\ Leite $^{* * * ;}$ Teresa Cristina Rangel Pereira****; Sergio Lins de-Azevedo-Vaz ${ }^{* * * * *}$
}

\footnotetext{
* Graduando em Odontologia da Universidade Federal do Espírito Santo

** Professora de Odontologia, Universidade Cruzeiro do Sul

*** Professora de Odontologia da União Metropolitana para o Desenvolvimento da Educação e Cultura

**** Professor(a) do Departamento de Clínica Odontológica da Universidade Federal do Espírito Santo
}

Recebido em 27/12/2016. Aprovado em 20/04/2017.

\begin{abstract}
RESUMO
A elaboração do diagnóstico correto e tratamento adequado é um dos maiores desafios que o cirurgião-dentista enfrenta na prática clínica. As radiografias são exames complementares neste processo, fornecendo informações importantes, e a radiografia panorâmica se destaca pela grande área examinada e pequena dose de radiação. O princípio da Justificação na Portaria SVS/MS no 453/98 afirma que os exames devem apresentar um motivo para exposição do paciente à radiação X. Objetivou-se analisar as prescrições de exames radiográficos por acadêmicos de Odontologia, encaminhadas ao Setor de Radiologia Odontológica da Universidade Federal do Espírito Santo (UFES) quanto às informações fornecidas e à justificação da exposição. Tratou-se de um estudo observacional transversal baseado em dados secundários. Foram utilizadas todas as solicitações de exames recebidos e executados no setor de Radiologia da UFES, no período de janeiro de 2013 a dezembro de 2015. Foram analisadas 1251 solicitações de exames radiográficos sendo que 1174 eram referentes a exames panorâmicos. Constatou-se que a maior parte dos pacientes era do gênero feminino, 48,5\% das solicitações não foram datadas, apenas 15,8\% possuíam registro da suspeita clínica, sendo que dessas 58,68\% traziam como justificativa "para fins de diagnóstico". Também foi observado que $92,1 \%$ estavam assinadas, 91,9\% foram carimbadas, 53,2\% das solicitações eram manuscritas e a disciplina que mais solicitou foi a Estomatologia. Em conclusão, ficou clara a necessidade de serem reforçados os conteúdos sobre a forma correta de se prescrever um exame complementar no curso de graduação.
\end{abstract}

Descritores: Radiografia. Diagnóstico Oral. Ensino Odontológico. 


\section{INTRODUÇÃO}

Desde a descoberta dos raios X em 1895, novos caminhos para o diagnóstico se abriram. Atualmente a radiografia é o exame complementar mais utilizado na prática odontológica ${ }^{1}$. Algumas técnicas são realizadas pelos próprios profissionais que estão realizando o atendimento clínico, enquanto outras, como a radiografia panorâmica, são realizadas, na maioria das vezes, em clínicas especializadas, devido à necessidade de equipamentos mais específicos.

Radiografias panorâmicas representam uma significativa parcela dos exames radiográficos realizados na prática clínica odontológica. Por meio delas é possível a observação e a análise dos dentes e suas alterações, assim como do complexo maxilomandibular ${ }^{2}$. A radiografia panorâmica possui extensa variedade de indicações que lhe conferem grande valor no diagnóstico e no acompanhamento do tratamento, além de algumas vantagens em relação ao exame periapical completo, como por exemplo, menor dose de radiação, menor custo para o paciente, maior área contemplada em uma única incidência com menor tempo de execução, além do aspecto legal, onde pode servir para atestar como era a situação do paciente antes do tratamento, ou avaliar o resultado final, servindo como prova legal, tanto para o cirurgião dentista quanto para o paciente ${ }^{3,4}$. Além disso, também pode fornecer dados à Odontologia Legal, responsável, junto com a Medicina Legal, pela identificação humana post-mortem, para auxiliar a identificação de corpos em vários estágios ${ }^{5}$.

Todo exame que emprega radiação $X$ traz consequências ao paciente, uma vez que a matéria, quando alvo de radiação, sofre ionização, na qual seus átomos tornam-se íons (positivos ou negativos). Assim, existe a possibilidade de efeitos biológicos determinísticos e estocásticos. Os primeiros geralmente só são observados quando doses altas de radiação são empregadas e causam a morte celular. Já os efeitos estocásticos, na maioria das vezes não são percebidos clinicamente e resultam em danos ao material genético que pode se tornar evidente em uma geração futura ${ }^{6}$. Além disso, destaca-se o seu efeito carcinogênico, como resultado das mutações genéticas promovidas nos tecidos irradiados ${ }^{7}$.

A Portaria 453, de 01 de junho de 1998, da Secretaria de Vigilância Sanitária do Ministério da Saúde $(\mathrm{SVS} / \mathrm{MS})^{8}$ é fundamentada no princípio ALARA (As Low As Reasonably Achievable) e afirma que o tempo de exposição deve ser o menor possível, o que justifica a realização de radiografias panorâmicas mediante a suspeita prévia de alguma patologia, de forma que o eventual dano da exposição à radiação seja inferior ao benefício recebido pelas informações da imagem obtida. A justificação para exposição é que ela deve ser realizada se vir a produzir benefícios suficientes para o indivíduo ou para a sociedade, para compensar os malefícios da radiação $X^{6}$.

Autores como Santos et al. ${ }^{9}$ e Gonçalves et $a l .{ }^{10}$, já demonstraram a importância de avaliar se as solicitações de exames efetuadas por cirurgiões-dentistas seguem esses princípios básicos ou se elas se tornaram uma ação banalizada devido à necessidade da rápida realização de procedimentos odontológicos rentáveis, ou da exigência de comprovação de execução de tratamento em pacientes para planos de saúde ${ }^{3,4}$. Por estar a instituição de ensino superior (IES), nesse cenário, como principal meio de formação dos futuros profissionais da área de saúde odontológica, justifica-se uma investigação para analisar se os princípios básicos da Portaria 453 estão sendo aplicados de maneira correta.

Objetivou-se analisar as prescrições de 
exames radiográficos encaminhadas ao Setor de Radiologia Odontológica da Universidade Federal do Espírito Santo (UFES), quanto às informações fornecidas pelo solicitante, bem como à justificação da exposição. Além disso, foram levantados dados para se traçar o perfil dos pacientes e dos exames solicitados.

\section{MATERIAIS E MÉTODOS}

Tratou-se de um estudo observacional com delineamento transversal baseado em dados secundários (solicitações de exames). Para o estudo, foram utilizadas todas as solicitações de exames realizados setor de Radiologia da UFES, no período de janeiro de 2013 a dezembro de 2015. Este estudo foi aprovado pelo Comitê de Ética em Pesquisa, parecer 849.847 e CAAE 37532814.0.0000.5060.

Os requerimentos foram analisados por dois estudantes do curso de graduação em Odontologia que já haviam cursado a disciplina de Radiologia, calibrados por dois professores dessa disciplina. Foram determinados os parâmetros de análise das solicitações como nome, idade, sexo, se a requisição foi manuscrita ou apenas assinalada em modelo impresso, os tipos de exames requisitados, se foi assinada por profissional registrado no Conselho Regional de Odontologia (CRO), se tinha o carimbo de um profissional registrado no $\mathrm{CRO}$, a disciplina de origem, se foi datada, se apresentava a suspeita clínica e, em caso positivo, a descrição da mesma. Algumas solicitações não estavam datadas e foi considerada a data em que o exame foi realizado.

As radiografias obtidas em função das requisições incluídas na amostra foram resgatadas dos prontuários eletrônicos para análise da confirmação, ou não, da suspeita clínica. Estas foram analisadas no próprio computador da disciplina de Radiologia, sem que quaisquer imagens fossem transferidas ou divulgadas. As dúvidas levantadas durante as etapas de coleta de dados, análise e interpretações radiográficas foram resolvidas por consenso entre os examinadores, e quando necessário, seus orientadores foram consultados.

Os dados foram inicialmente analisados por meio de estatísticas descritivas. Para avaliar se as solicitações estavam de acordo com a Portaria SVS/MS n ${ }^{\circ} 453$, de 1 de junho de $1998^{4}$, foi utilizado $o$ teste qui-quadrado para comparação de proporções. O nível alfa de significância foi adotado em $1 \%$ com intervalo de confiança de $95 \%$. O programa utilizado nas análises foi IBM SPSS Statistics version 21.

\section{RESULTADOS}

Um total de 1251 solicitações recebidas pela Clínica de Radiologia da UFES foram resgatadas para o estudo.

Do total dos pacientes atendidos, 62,8\% eram do sexo feminino. Em 14 solicitações o sexo do paciente não pôde ser identificado com base no nome do paciente, ou por grafia incompreensível. Em mais de $99 \%$ das solicitações analisadas, a idade do paciente não estava registrada e, portanto, não foi possível definir o perfil etário dos pacientes que são atendidos na clínica de radiologia, somente utilizando a solicitação como fonte de informação.

Quanto ao tipo de apresentação da solicitação, a mais usual foi a forma manuscrita, com 53,2\% ( $\mathrm{n}=666)$ (tabela 1). A disciplina de Estomatologia foi a que mais solicitou o exame complementar radiográfico, correspondendo a 28,5\% $(\mathrm{n}=356)$ dos pedidos analisados (tabela 1). Ao todo, 1174 exames relacionavam-se à radiografia panorâmica, associada ou não a outros exames, contabilizando $92,4 \%$ das indicações.

Quanto à identificação do solicitante, 92,1\% $(n=1152)$ das requisições foram assinadas e $91,9 \%$ 
( $\mathrm{n}=1150$ ) carimbadas, mas também foi observado que $2,6 \%(n=33)$ das solicitações carimbadas pela disciplina solicitante estavam sem o número do registro no $\mathrm{CRO}$, e $2,1 \%$ das solicitações $(\mathrm{n}=26)$ não apresentavam o carimbo, somente o registro por escrito do CRO do dentista responsável. Além disso, 48,5\% ( $\mathrm{n}=607)$ das solicitações não foram datadas (tabela 2).

A solicitação de exame mais recorrente na prática odontológica da UFES foi a panorâmica sem complementação, correspondendo a 82,6\% ( $n=1034)$, seguida da demanda de radiografia panorâmica associada a outra técnica radiográfica com $11,7 \%(\mathrm{n}=146)$ (tabela 3$)$.
Em relação à indicação da suspeita clínica, foi observado que somente $15,8 \%(n=198)$ das solicitações a continham, e destas, as indicações mais frequentes foram aquelas com fins cirúrgicos com 40,4\% ( $\mathrm{n}=80)$. Evidenciamos na amostra 258 solicitações com os dizeres "para fins de diagnóstico", termo genérico e subjetivo que não contempla uma indicação correta.

Quando confrontamos as imagens com a suspeita clínica, observou-se a coerência entre estas em 89,4\% $(\mathrm{n}=177)$ dos casos, e destes, $73,7 \%(\mathrm{n}=146)$ foi solicitado o exame radiográfico mais adequado de acordo com o indicado como hipótese diagnóstica (tabela 4).

Tabela 1. Tipo de solicitação utilizada e disciplina de origem

\begin{tabular}{llcc}
\hline Variável & & $\mathbf{n}$ & $\mathbf{\%}$ \\
\hline \multirow{2}{*}{ Tipo } & Redigida & 666 & 53,2 \\
& Assinalada & 585 & 46,8 \\
\hline \multirow{5}{*}{ Disciplina } & Estomatologia & 356 & 28,5 \\
& Cirurgia II & 337 & 26,9 \\
& Cirurgia I & 132 & 10,6 \\
& Odontopediatria & 118 & 9,4 \\
& Periodontia & 86 & 6,9 \\
& Clínica Integrada & 68 & 5,4 \\
& Núcleo de Diagnóstico Bucal & 62 & 5,0 \\
& Ortodontia & 37 & 3,0 \\
& Odontologia Restauradora Clínica & 12 & 1,0 \\
& Prótese & 7 & 0,6 \\
& Clínica Integrada de Atenção Primária & 3 & 0,2 \\
& Endodontia & 2 & 0,2 \\
& Sem informação & 31 & 2,5 \\
\hline
\end{tabular}

Tabela 2- Identificação profissional e data registrada nas solicitações

\begin{tabular}{llcc}
\hline Variável & & n & \% \\
\hline \multirow{2}{*}{ Assinada } & Não & 99 & 7,9 \\
& Sim & 1152 & 92,1 \\
\hline \multirow{4}{*}{ Carimbada } & Não & 75 & 6,0 \\
& Não, mas com CRO & 26 & 2,1 \\
& Sim & 1117 & 89,3 \\
& Sim, mas sem CRO & 33 & 2,6 \\
\hline \multirow{2}{*}{ Datada } & Não & 607 & 48,5 \\
& Sim & 644 & 51,5 \\
\hline
\end{tabular}


Tabela 3. Comparação entre tipo de exame e presença da suspeita clínica.

\begin{tabular}{lcccc}
\hline Radiografia & $\begin{array}{c}\text { Sem suspeita clínica } \\
\text { n }(\boldsymbol{\%})\end{array}$ & $\begin{array}{c}\text { Com suspeita clínica } \\
\mathbf{n}(\boldsymbol{\%})\end{array}$ & & \\
\hline Panorâmica & $869(84,05)$ & $165(15,95)$ & $\mathrm{a}$ & \\
Intrabucais & $35(59,32)$ & $24(40,68)$ & $\mathrm{b}$ & $\mathrm{X}^{2}=33.9667$ \\
Extrabucais & $8(66,67)$ & $4(33,34)$ & $\mathrm{ab}$ & \\
Panorâmica + outra & $134(91,78)$ & $12(8,22)$ & $\mathrm{c}$ & $\mathrm{p}<0.01^{*}$ \\
Total & $1046(100,00)$ & $205(100,00)$ & & \\
\hline
\end{tabular}

a. Comparável ao grupo "Panorâmica" (proporção mediana de exames sem suspeita)

b. Comparável ao grupo "Intrabucais" (menor proporção de exames sem suspeita)

c. Comparável ao grupo "Panorâmica + outro" (maior proporção de exames sem suspeita)

* Estatisticamente significante ao nível de 1\% - Teste Qui-quadrado.

Tabela 4. Análise da presença de suspeita clínica, indicação, coerência com o encontrado na imagem e verificação do exame mais adequado.

\begin{tabular}{llcc}
\hline Variável & & $\mathbf{n}$ & $\mathbf{\%}$ \\
\hline \multirow{2}{*}{ Suspeita Clínica } & Não & 1053 & 84,2 \\
& Sim & 198 & 15,8 \\
\hline \multirow{4}{*}{ Indicação } & Fins cirúrgicos & 80 & 40,4 \\
& Fins ortodônticos & 12 & 6,1 \\
& Informação diagnóstica & 58 & 29,3 \\
& Laudo odontológico & 2 & 1,0 \\
& Planejamento implante & 5 & 2,5 \\
& Planejamento protético & 5 & 2,5 \\
& Tratamento periodontal & 36 & 18,2 \\
& Não & 21 & 10,6 \\
\multirow{2}{*}{ Adequêcia } & Sim & 177 & 89,4 \\
& Não & 52 & 26,3 \\
& Sim & 146 & 73,7 \\
\hline
\end{tabular}

\section{Discussão}

As Instituições de Ensino Superior têm papel fundamental na formação dos futuros profissionais de Odontologia, o que inclui a instrução da prescrição dos exames radiográficos e interpretação dos mesmos ${ }^{11,12}$. Neste sentido, observou-se que a maioria das solicitações radiográficas se apresentava preenchida de forma incompleta, com ausência de dados importantes. Este é um fator relevante quando se remete às competências gerais das Diretrizes Curriculares em Odontologia (DCN), em que as instituições de ensino devem contemplar os conhecimentos para que o futuro profissional possa tomar 
decisões fundamentadas em condutas sistematizadas e baseadas em evidências científicas $^{13}$. Assim, cabe à instituição promover medidas corretivas, tendo em vista a importância do seu papel na formação profissional, pois é nessa fase que são adquiridos alguns hábitos dos futuros profissionais.

A maioria das solicitações radiográficas foi feita para pacientes do sexo feminino, concordando com os achados de Gonçalves et $a l .{ }^{10}$. Porém não foi possível obter dados suficientes, nas solicitações, para determinar a idade daqueles que procuraram a clínica de radiologia para realização de seu exame.

Uma grande quantidade das solicitações recebidas pelo setor de Radiologia da UFES foi feito em um modelo digitado padrão (46,8\%), no qual o solicitante apenas assinala qual ou quais exames deveriam ser realizados. Tal modelo impresso é distribuído inclusive por clínicas de Radiologia Odontológica privadas, com a finalidade de facilitar a solicitação de exames e, ainda, como uma forma de publicidade. Apesar do cirurgião-dentista ganhar tempo de trabalho sem ter a necessidade de preencher por escrito a requisição, uma vez o requerimento assinado e carimbado, o próprio paciente ou outros indivíduos podem, de má fé ou até mesmo inocentemente, assinalar diversas outras opções de exames e serem expostos desnecessariamente à radiação $\mathrm{X}$.

A grande maioria dos encaminhamentos utilizados no estudo estavam assinados $(92,1 \%)$ e carimbados (91,9\%). Contudo, esses valores deveriam ser integrais, uma vez que, para fazer solicitação de um exame que traz riscos ao paciente, o cirurgião dentista deve incluir no requerimento seu carimbo, número de $\mathrm{CRO}$ e assinatura (Portaria SVS/MS 453/98) ${ }^{8}$. No estudo de Oswall et al. em $2009^{14}$, a maioria das solicitações não foram carimbadas, sendo esse o campo menos preenchido no estudo. Em algumas situações essa se torna a única forma de identificar o solicitante, já que a assinatura não é um meio eficaz de identificação. A falta desses parâmetros compromete não somente a clínica de Radiologia por realizar exames sem o respaldo de que foram realmente solicitados pelo dentista, mas também o próprio paciente que pode se deparar com a situação de não poder realizar o exame, caso esta solicitação seja negada pelo responsável pela execução do exame.

Apesar de estudos como o de Gonçalves et al. ${ }^{10}$ demonstrarem que a maioria das solicitações recebidas tem origem de demandas ortodônticas, no presente estudo, constatou-se que a disciplina de Estomatologia foi a que deteve esse posto. Isso pode ser explicado pelo fato de professores de Radiologia também atuarem nessa disciplina, bem como o fato de que os pacientes atendidos e que apresentem alguma queixa que indique alterações nas estruturas ósseas e/ou dentárias sejam encaminhados para realização do exame radiográfico panorâmico, acompanhado ou não por outros exames complementares.

Autores como Jonhson ${ }^{15}$ e Castilho et al. ${ }^{16}$ defendem em seus estudos que a radiografia panorâmica deve ser sempre realizada, devido a quantidade de informações fornecidas e muitas vezes não obtidas somente com exames clínicos. C0ntudo, é contestada por vários autores ${ }^{17-19}$ que condenam seu uso como exame de rotina, alegando que a quantidade de lesões diagnosticadas somente por meio desse exame é mínima, o que está de acordo com os princípios já mencionados de otimização de dose e redução de exposição ao paciente.

De todas as solicitações recebidas e analisadas, apenas 15,8\% apresentaram alguma forma de justificativa quanto à suspeita da condição do paciente. Fazer um requerimento de um exame radiográfico sem uma justificativa por si só já é o bastante para não atender aos mínimos requisitos de uma correta solicitação de exame. 
Além disso, 58,6\% desses poucos exames que foram "justificados" traziam no corpo do requerimento a seguinte indicação "para fins de diagnóstico',. O termo per se não está errado, uma vez que esta é a finalidade do exame radiográfico, porém é extremamente amplo e não deixa explícita qual a área de interesse para o radiologista ou técnico em radiologia. Apesar de extremamente alta a quantidade de solicitações sem a suspeita clínica, pode-se presumir que nem sempre isso ocorra por falta de conhecimento do profissional ou ausência de abordagem pedagógica sobre a temática para os graduandos, já que os docentes da área abordam esse assunto. Salienta-se que os modelos impressos para serem assinalados não trazem um campo para que seja preenchida tal indicação e o pequeno espaço para "observações" normalmente não é suficiente para descrever a suspeita do que acomete o paciente.

Apesar de todas as desvantagens apresentadas, o modelo impresso estava em vigor na IES pesquisada, com grande estoque de cópias de seus formulários. Além disso, as próprias clínicas particulares de radiologia distribuem solicitações desse tipo, que acabam chegando até o setor de radiologia da UFES.

Das solicitações analisadas, 14 não puderam ser identificadas quanto ao sexo do paciente, ou pelo nome ambíguo ou pela grafia incompreensível. Em mais de 99\% das solicitações analisadas, a idade do paciente não estava redigida e não nos permitiu definir o perfil etário dos pacientes que foram atendidos na clínica de radiologia o que poderia auxiliar ao radiologista na elaboração do diagnóstico de possíveis alterações encontradas. O Royal College of Radiologists (RCR) que emite periodicamente orientações sobre assuntos voltados para a Radiologia, afirma que as solicitações devem ser feitas com precisão e de forma legível para evitar qualquer má interpretação, devem conter o motivo pelo qual o exame é solicitado ${ }^{14}$.

A importância da comunicação entre o solicitante e o profissional de Radiologia que executará o exame foi ressaltada por Stavem et $a l .{ }^{20}$. A precisão de informações influencia diretamente na qualidade do exame. Para que isso ocorra é importante que sejam desenvolvidos meios para que essa troca de informações seja mais precisa. Novamente destaca-se a necessidade de se desenvolver nos acadêmicos as competências descritas nas DCN quanto a comunicação, onde a habilidade da escrita deve ser estimulada para se manter a confidencialidade das informações obtidas em confiança, na interação com outros profissionais de saúde e com o público em geral ${ }^{13}$.

Segundo a análise estatística quanto aos exames que foram encaminhados com registro da suspeita clínica, $89,4 \%$ desses tiveram observações que justificassem a realização do exame, mostrando que as informações coletadas no exame clínico do paciente resultaram na correta indicação da técnica radiográfica a ser realizada para confirmação do diagnóstico e tratamento do paciente. Já em relação ao tipo de exame requisitado, $73,7 \%$ das solicitações correspondiam à melhor técnica para observação da suspeita relatada. Isso mostra que os acadêmicos e os professores estão requisitando exames adequadamente, pois ao realizar a técnica correta, evita-se que o paciente tenha que ser submetido a outros exames complementares.

As solicitações preenchidas inadequadamente podem ocasionar problemas legais e principalmente prejuízos com relação à saúde do paciente $^{21}$. Bosmans et al. ${ }^{22}$, afirmaram que é ilusória a existência de uma solicitação de radiografia perfeitamente estruturada. Seria interessante, portanto um modelo padrão para que a discrepância de informações de um lugar 
para outro não seja tão expressiva como apontam os estudos.

O impacto da padronização e informatização das solicitações radiográficas foi avaliado e demonstrou que essa padronização resultou em acentuada melhoria da qualidade dos dados necessários para realização do exame, além do aumento da satisfação do solicitante, pois o exame era realizado de forma adequada e com as informações necessárias ${ }^{23}$. Seguindo essa linha de raciocínio, foi criado pelos acadêmicos envolvidos nessa pesquisa, um padrão de solicitação e esse implementado para todas as disciplinas na IES objetivo desse estudo (figura 1).

\section{PAPEL TIMBRADO DA INSTITUIÇÃO \\ SOLICITAÇÃO}

Solicito a realização de radiografia(s) TIPO DE RADIOGRAFIA (PANORÂMICA / PA / TELERRADIOGRAFIA LATERAL...) para o paciente NOME COMPLETO DO PACIENTE, GÊNERO E IDADE, para investigação de SUSPEITA OU INDICAÇÃO CLÍNICA.

LOCAL, DATA

\section{CARIMBO E ASSINATURA DE UM PROFISSIONAL RESPONSÁVEL}

Figura 1. Modelo proposto para utilização na IES

\section{CONCLUSÃO}

A partir dos resultados obtidos fica clara a necessidade de serem reforçados os ensinamentos sobre a forma correta de se solicitar um exame complementar, seja ele radiográfico ou não, e a importância de descontinuar o uso de modelos impressos assinaláveis como forma de pedido de exames. Assim, os autores propõem que seja criado um modelo de requerimento a ser adotado que traga campos pré-determinados a serem preenchidos, de tal forma que o solicitante não se esqueça de nenhum dado. É necessário que as informações nas solicitações sejam escritas por extenso, não havendo a possibilidade de serem alteradas posteriormente por quaisquer motivos. É importante ressaltar que o requerimento tenha espaço suficiente para que o solicitante redija tudo o que necessita ser informado ao profissional que irá realizar o exame. 


\begin{abstract}
Analysis of the radiographic requests used by dental students of a Brazilian Public University and the development of a didactic model
\end{abstract}

The development of a correct diagnosis and an effective treatment is one of the biggest challenges that the dentist must face on the clinic. The panoramic radiography is a complementary exam used on the stands out for the large area examined and a small radiation dose. The Justification principle on the Ordinance SVS/MS $\mathrm{n}^{\circ} 453 / 98$ states that the exams must have a reason to expose the patient to $\mathrm{X}$-ray radiation. The objective of this study was to analyze the radiographic requests sent to the Radiology Division of Universidade Federal do Espírito Santo (UFES), searching for the information given by the requester, as well as the justification of the exposure. It was a transversal study based on secondary data. All requests for exams received and executed by the Radiology Department of UFES, between January 2013 and December 2015, were used. A total of 1251 requests were analyzed in which 1174 referred to panoramic exams. Most of the patients were female, $48.5 \%$ did not have a date, only $15,8 \%$ had a clinic indication and $58.68 \%$ of these brought as justification "for diagnosis". Also, were observed that $92,1 \%$ were singed and 91,9\% stamped, $53.2 \%$ were handwritten and came mostly from the Stomalogy clinic. In conclusion, it was clear the need to reinforce the teachings about the correct way to request complementary exams in the undergraduate course.

Descriptors: Panoramic Radiography. Oral Diagnostic. Radiography.

\section{REFERÊNCIAS}

1. Tosoni G, Campos D, Silva M. Frequência de cirurgiões-dentistas que realizam exame radiográfico intrabucal e avaliação das condições para a qualidade do exame. Rev Odontol Unesp. 2003;32(1):25-9.

2. Coldebella JF. Aplicabilidade da Radiografia Panorâmica [dissertação]. Curitiba: Universidade Tuiuti do Paraná; 2011.
3. Gonçalves PE, Dotta EAV, Serra M da C. Imageologia na odontologia e aspectos legais. RGO. 2011;59(suplemento 0):89-95.

4. Carvalho P, Lopes A, Silva C, Assis A, Medeiros J. Utilização de protocolo radiográfico na clínica de Odontopediatria. Rev Bras Odontol. 2010;67(2):279-82.

5. Carvalho SPM, Silva RHA, Lopes-Júnior C, Peres AS. A utilização de imagens na identificação humana em odontologia legal. Radiol Bras. 2009;42(2):125-30.

6. Engel-Hills P. Radiation protection in medical imaging. Radiography. 2006;12(2) :153-60.

7. ICRP, 2012. ICRP Statement on Tissue Reactions / Early and Late Effects of Radiation in Normal Tissues and Organs Threshold Doses for Tissue Reactions in a Radiation Protection Context. ICRP Publication 118. Ann. ICRP 41(1/2).

8. Brasil. Ministério da Saúde. Secretaria de Vigilância Sanitária. Diretrizes de Proteção Radiológica em Radiodiagnóstico Médico e Odontológico. Portaria $\mathrm{N}^{\mathrm{o}} 453$, de $1^{\circ}$ de junho de 1998. [Acesso em 12 dez. 2016]. Disponível em: http://www.cro-rj.org.br/ $\underline{\text { radiologia/PF453integra.pdf }}$

9. Santos KCP, Oliveira AS, Hesse D, Buscatti MY, Oliveira JX. Avaliação de radiografias panorâmicas objetivando o cotejamento entre os motivos da solicitação e eventuais achados radiográficos. Rev Inst Cienc Saúde. 2007;25(4):419-22.

10. Gonçalves A, Gonçalves M, Bóscolo FN. Avaliação das solicitações de radiografias recebidas por clínica de radiologia odontológica. RFO UPF. 2003;8(1):55-61.

11. Kantor ML, Slome BA. The effect of eliminating administrative radiographs on patient exposure and accuracy of provisional treatment plans. J Dent Educ. 1987;51 (12):701-4. 
12. Bohay R, Stephens R, Kogon S. A survey of the radiographic practices of general dentists for edentulous patients. Oral Surg Oral Med Oral Pathol Oral Radiol. 1995;79(3):526-31.

13. Ministério da Educação. Conselho Nacional de Educação Câmara de Educação Superior. Resolução CNE/CES 3, de 19 de fevereiro de 2002. Institui Diretrizes Curriculares Nacionais do Curso de Graduação em Odontologia. Diario Oficial Uniao. 04 mar 2002; Seção1:10. [Acesso em 22 set. 2016]. Disponível em: http://portal.mec.gov.br/ cne/arquivos/pdf/ CES032002.pdf

14. Oswal D, Sapherson D, Rehman A. A study of adequacy of completion of radiology request forms. Radiography. 2009;15(3) :209-13.

15. Johnson C. Analysis of panoramic survey. J Am Dent Assoc. 1970;81:151-4.

16. Castilho JCM, Armond MC, Médici Filho E, Moraes MEL. Avaliação de "achados radiográficos" em pacientes encaminhados para tratamento ortodôntico e/ou ortopédico facial. J Bras Ortodon Ortop Facial. 2001; 6(35):422-9.

17. Stephens RG, Kogon SL, Speechley MR, Dunn WJ. A Critical View of the rationale for routine, initial and periodic radiographic surveys. J Can Dent Assoc. 1992;58(10) :825-37.

18. Rushton VE, Horner K, Worthington H V. Screening panoramic radiology of adults in general dental practice: radiological findings. Br Dent J. 2001;190(9):495-501.
19. Mendes FM, Novaes TF, Matos R, Gimenez T, Imparato JCP, Braga MM. Métodos complementares na detecção de lesões de cárie em dentes decíduos são realmente necessários? Rev Assoc Paul Cir Dent. 2014;68(1):54-9.

20. Stavem K, Foss T, Botnmark O, Andersen OK, Erikssen J. Inter-observer agreement in audit of quality of radiology requests and reports. Clin Radiol. 2004;59(11):1018-24.

21. Triantopoulou C, Tsalafoutas I, Maniatis P, Papavdis D, Raios G, Siafas I, et al. Analysis of radiological examination request forms in conjunction with justification of X-ray exposures. Eur J Radiol. 2005;53(2):306-11.

22. Bosmans JML, Weyler JJ, Parizel PM. Structure and content of radiology reports, a quantitative and qualitative study in eight medical centers. Eur J Radiol [Internet]. 2009;72(2):354-8.

23. Troude P, Dozol A, Soyer P, Girard D, Martinez F, Montagne B, et al. Improvement of radiology requisition. Diagn Interv Imaging. 2014;95(1):69-75.

Correspondência para:

Rafael Mendes Del Queiroz

e-mail: rafael.mdq@ hotmail.com

Universidade Federal do Espírito Santo

Rua Guanabara, 1542 Itapoã

29101-592 Vila Velha/ES 